\title{
PROMOTIONSKOLLEG: PSYCHOSOZIALE FOLGEN VON MIGRATION UND FLUCHT - GENERATIONALE DYNAMIKEN UND ADOLESZENTE VERLÄUFE
}

Susanne Benzel \& Vera King

Sigmund-Freud-Institut (SFI)

E-Mail: benzel@sigmund-freud-institut.de

URL: https://www.sigmund-freud-institut.de/index.php/mitarbeiter-innen/dr-susannebenzell

Goethe-Universität Frankfurt, Institut für Soziologie

E-Mail: king@soz.uni-frankfurt.de

URL: https://www.fb03.uni-frankfurt.de/45643896/Prof_Dr_Vera_King

Zitationsvorschlag:

Benzel, Susanne/King, Vera (2020): Promotionskolleg: Psychosoziale Folgen von Migration und Flucht - generationale Dynamiken und adoleszente Verläufe. In: Gesellschaft - Individuum - Sozialisation (GISO). Zeitschrift für Sozialisationsforschung, 1 (2). DOI: 10.26043/GISo.2020.2.6

Link zum Artikel:

https://doi.org/10.26043/GISo.2020.2.6 


\title{
PROMOTIONSKOLLEG: PSYCHOSOZIALE FOLGEN VON MIGRATION UND FLUCHT - GENERATIONALE DYNAMIKEN UND ADOLESZENTE VERLÄUFE
}

\author{
Susanne Benzel \& Vera King \\ Promotionskolleg am Sigmund-Freud-Institut (SFI) \\ Gefördert von der Hans-Böckler-Stiftung \\ Laufzeit: 12/2018-11/2021 \\ Leitung: \\ Prof. Dr. Vera King (Sprecherin), SFI, Goethe-Universität Frankfurt \\ Prof. Dr. Hans-Christoph Koller, Universität Hamburg \\ Prof. Dr. Patrick Meurs, SFI, Universität Kassel \\ Prof. Dr. Heinz Weiß, SFI, Robert-Bosch-Krankenhaus Stuttgart, Universität Tübingen
}

Koordination: Dr. Susanne Benzel, SFI

Promotionsstipendiat*innen: Irina Dannert, Franziska Heinz, Simon Heyny, Ina Kulić, Alexandra Litinskaya, Maximilian Richter, Tassilo Schuster, Hannes Weidmann, Luisa Wirth

Homepage: www.promotionskolleg-migration.de

\section{ZIELE UND AUSRICHTUNG DES PROMO- TIONSKOLLEGS}

Das Promotionskolleg zielt auf die interdisziplinäre Erforschung der psychosozialen und psychischen Folgen von Migration und Flucht und deren Verarbeitung und Bewältigung angesichts prekärer gesellschaftlicher Rahmenbedingungen. Da Migration und Flucht oftmals Projekte sind, die die gesamte Familie betreffen, richtet sich der Blick auf die Eltern-Kind-Beziehungen und Generationendynamiken - im Besonderen auf die Formen transgenerationaler Weitergabe des familialen oder elterlichen ,Erbes' oder der biographischen Umgestaltungen in der Adoleszenz. Um Mechanismen transgenerationaler Weitergabe der psychosozialen Migrationsfolgen für die Kindergenerationen sowohl in den produktiven als auch in den einschränkenden Dimensionen zu erforschen, werden typische Konstellationen und Bewältigungsmuster, ihre Merkmale, Widersprüche und Ambivalenzen im Rahmen qualitativ-rekonstruktiver Zugänge herausgearbeitet. Im Zentrum stehen adoleszente Generationendynamiken im Kontext von Flucht und Migration, die durch die interdisziplinären Verknüpfungen von Soziologie/Sozialpsychologie, erziehungswissenschaftlicher Erforschung von Bildungsbiographien, Kinder- und Jugendpsychologie sowie psychoanalytischer Psychosomatik beleuchtet werden. Das Promotionskolleg beinhaltet vier Forschungsschwerpunkte, die im Folgenden kurz beleuchtet werden.

PSYCHOANALYTISCH-SOZIALPSYCHOLOGISCHE UND SOZIOLOGISCHE PERSPEKTIVE (KING)

Im Schwerpunkt I stehen psychosoziale und psychodynamische Bedeutungen von kulturellen und religiösen Selbstverortungen junger Frauen und Männer im Fokus. Insbesondere in der Adoleszenz können für die Bewältigung von transgenerational vermittelten Erfahrungen der Migration und Flucht das Aufgreifen dieser Orientierungen integrative Lösungen darstellen, aber auch rigide Abwehrkonstrukte, gerade wenn Religion und Kultur starr ausgelegt werden (King 2016). Daran anknüpfend geht Ina Kulić in 
ihrer Promotion der Frage nach der psychodynamischen und sozialen Funktion von kulturellen Deutungsmustern bei der Bewältigung und Verarbeitung von fluchttypischen Herausforderungen am Beispiel von geflüchteten jungen Frauen aus dem ehemaligen Jugoslawien nach. Hannes Weidmann wiederum analysiert in seiner Dissertation insbesondere die psychosoziale Bedeutung von muslimischer Religiosität am Beispiel junger Männer der 3. Generation aus türkischstämmigen Familien. Hierbei werden auch intergenerationale Dynamiken im Hinblick auf Männlichkeitsentwürfe sowie (strukturelle) Ausgrenzungs- und Diskriminierungserfahrungen in den Blick genommen. In welcher Weise adoleszente Identitätsfragen im Kontext von Migrationserfahrungen in Selbstdarstellungen in den sozialen Medien zum Ausdruck gebracht werden und welche Bedeutung diese wiederum für die Beziehungsgestaltung von an- und abwesenden Bezugspersonen sowie der Verbindung zum Herkunftsland und anderen Lebensorten einnehmen, wird von Susanne Benzel in Rahmen ihres Habilitationsprojekts untersucht.

\section{ERZIEHUNGSWISSENSCHAFTLICHE PER- SPEKTIVE (KOLLER)}

Im Schwerpunkt II wird der Frage nachgegangen, welche Folgen Migration für die Bildungsprozesse Jugendlicher und junger Erwachsener hat. Dabei kommt den adoleszenten Ablösungsprozessen und der intergenerationalen Familiendynamik eine entscheidende Bedeutung zu, da sowohl formale Bildungskarrieren als auch biographische Bildungsprozesse in einem engen Zusammenhang mit dem Verlauf der adoleszenten Umgestaltung des Generationenverhältnisses stehen (Koller 2012). Im Anschluss an bisherige Befunde zweier Forschungsprojekte zu jungen Männern aus türkischen und italienischen Migrantenfamilien (King/Koller 2009) nehmen Franziska Heinz und Tassilo Schuster in ihren Dissertationen junge Frauen aus türkischen und junge Männer aus vietnamesischen Migrantenfamilien in den Blick - beides Gruppen, welche im Vergleich durchschnittlich als bildungserfolgreich gelten. Von Bedeutung sind dabei einerseits potenzielle geschlechtertypische Muster adoleszenter Ablösung von den Eltern und andererseits eine Rückbindung an Diskurse über das sogenannte asiatische Wunder.

\section{ENTWICKLUNGSPSYCHOLOGISCHE SOWIE KINDER- UND JUGENDANALYTISCHE PER- SPEKTIVE (MEURS)}

Im Schwerpunkt III werden offene Fragen zu psychosozialen Entwicklungen von unbegleiteten und begleiteten minderjährigen Geflüchteten untersucht. Hierbei stehen besonders die Bewältigung der adoleszenten Veränderungen angesichts erhöhter Unsicherheiten, Vulnerabilitäten und Krisenpotenziale im Vordergrund. Irina Dannert und Maximilian Richter erforschen in ihren Dissertationen mittels Analysen narrativer Interviews insbesondere die Identitäts- und Bindungssuche in den spezifischen Konstellationen von an- und abwesenden Eltern sowie Bezugspersonen. Die Erkenntnisse münden in Empfehlungen für präventive, begleitende Maßnahmen für Adoleszente und ihre Eltern in Weiterentwicklung von Ansätzen, wie sie Meurs/Jullian (2016) zu Migrantenfamilien mit Kleinkindern entworfen haben.

\section{MEDIZINISCH-PSYCHOSOMATISCHE UND KLINISCH-PSYCHOANALYTISCHE PERSPEK- TIVE (WEIß)}

Im Schwerpunkt IV werden vor allem die spezifischen psychischen Vulnerabilitäten und leiderzeugenden Potenziale im Kontext von Migration sowie intergenerationale Dimensionen und unterschiedlich produktive oder einschränkende Formen der Bewältigung bis hin zu psychischen Erkrankungen in adoleszenten Entwicklungen untersucht. Alexandra Litinskaya fokussiert in diesem Kontext in ihrer Forschung das Familienbild bei Patient*innen, verbunden mit der Frage, in welcher Weise Generationendynamiken und Migrationserfahrungen mit psychischen und psychosomatischen Erkrankungen zusammenhängen. Luisa Wirth untersucht wiederum die subjektiven Krankheitstheorien im Zusammenhang mit Migrationserfahrungen bei Patient*innen mit einer Borderline-Persönlichkeitsproblematik. In beiden Forschungen werden äuBere Umstände in der Ankunftsgesellschaft, die Leiden potenzieren oder abmildern können, in die Analyse einbezogen und mit klinisch-diagnostischen Fragen verknüpft (Weiß 2017). 
Der innovative Gewinn dieses viergliedrigen Forschungsansatzes liegt insgesamt in der Möglichkeit, den in vielerlei Hinsicht noch offenen Fragen zur komplexen generationalen Vermittlung im Verhältnis zu den adoleszenten Entwicklungsprozessen im Migrationskontext nachgehen zu können. Eine übergreifend qualitativ-rekonstruktive - auch psychodynamische Prozesse erschließende - Orientierung ermöglicht es, latent wirksame Strukturen und Anpassungsmechanismen, produktiv-kreative und destruktive Potenziale sowie die psychosozialen Kosten von Ausgrenzung und Diskriminierung präzise herauszuarbeiten, sodass psychosoziale Migrationsfolgen in ihrer Vielschichtigkeit rekonstruiert werden können. In dieser grundlagentheoretischen Hinsicht bearbeitet Simon Heyny in seiner Promotion die Frage, inwiefern sich anhand psychischen Leidens Gesellschaftskritik formulieren lässt.

\section{LITERATUR}

King, Vera (2016): Zur Psychodynamik von Migration. Muster transgenerationaler Weitergabe und ihre Folgen in der Adoleszenz. In: Zeitschrift für Psychoanalyse und ihre Anwendungen, 70, 977-1002.

King, Vera/Koller, Hans-Christoph (Hrsg.) (2009): Adoleszenz - Migration - Bildung. Bildungsprozesse Jugendlicher und junger Erwachsener mit Migrationshintergrund. 2. erweiterte Auflage, Wiesbaden: VS-Verlag für Sozialwissenschaften.

Koller, Hans-Christoph (2012): Bildung anders denken. Einführung in die Theorie transformatorischer Bildungsprozesse. Stuttgart: Verlag W. Kohlhammer.

Meurs, Patrick/Jullian, Gül (2016): Entwicklungsberatung für Familien mit Kleinkindern aus verschiedenen Migrationswellen. In: BurkhardtMußmann, Claudia/Dammasch Frank (Hrsg.): Migration, Flucht und Kindesentwicklung. Frankfurt: Brandes \& Apsel Verlag, 14-25.

Weiß, Heinz (2017): Trauma, Schuldgefühl und Wiedergutmachung. Affekte, die innere Entwicklung ermöglichen. Stuttgart: Klett-Cotta.

\section{Zu den Autor*innen}

Susanne Benzel, Dr. phil., Diplom in Erziehungswissenschaft, ist wissenschaftliche Mitarbeiterin am Sigmund-Freud-Institut; zuvor Vertretungsprofessorin an der Goethe-Universität Frankfurt am Institut für Soziologie, Fachbereich Gesellschaftswissenschaften. Ihre Forschungsschwerpunkteliegen in den Bereichen Adoleszenz-, Jugend- und Familienforschung, Migration und Flucht, psychische und soziale Folgen von Digitalisierung, Generations- und Biografieforschung sowie Methoden qualitativer Sozialforschung.

Vera King, Prof. Dr. phil., ist Professorin für Soziologie und psychoanalytische Sozialpsychologie an der Goethe-Universität Frankfurt und Direktorin des Sigmund-Freud-Instituts. Zuvor war sie Professorin für Sozialisations- und Entwicklungsforschung an der Universität Hamburg. Ihre Forschungsschwerpunkte sind Studien zu Folgen gesellschaftlichen Wandels, zum Verhältnis von Kultur und Psyche, insbesondere im Kontext von Beschleunigung, Optimierung und Digitalisierung, Theorie- und Forschungsprojekte zu Generativität und Generationenbeziehungen, Kindheit, Jugend/Adoleszenz und Wandel von Familien. 


\section{Kontakt}

Dr. Susanne Benzel

Sigmund-Freud-Institut

Myliusstraße 20

D-60323 Frankfurt am Main

Tel.: +4969971204116

E-Mail: benzel@sigmund-freud-institut.de

URL: https://www.sigmund-freud-institut.de/index.php/mitarbeiter-innen/dr-susanne-benzel/

Prof. Dr. Vera King

Institut für Soziologie - Fachbereich Gesellschaftswissenschaften

Goethe-Universität Frankfurt am Main

Theodor-W.-Adorno-Platz 6

D-60323 Frankfurt/M.

Sekretariat: Frau Charlesworth

E-Mail: charlesworth@soz.uni-frankfurt.de 